\title{
氨基二硫代甲酸酯类化合物的合成及生物活性研究进展
}

\author{
李日东王元强葛泽梅李润涛* \\ (北京大学药学院 天然药物及仿生药物国家重点实验室 北京 100191)
}

\begin{abstract}
摘要 氨基二硫代甲酸酯类化合物由于具有广泛的生物活性, 其合成方法和药理活性的研究已成为有机化学和药物化 学领域研究的热点. 在合成方法方面, 对在强碱条件下、弱碱条件下、以水为溶剂、以离子液为溶剂和无溶剂条件下 合成该类化合物的方法进行了综述. 在生物活性方面，对该类化合物在治疗慢性酒精中毒、抗氧化、抗病毒、抗菌、 抑制胆碱酯酶、肿瘤预防以及抗肿瘤方面的作用进行了综述
\end{abstract}

关键词＼cjkstart氨基二硫代甲酸酯; 合成; 生物活性; 研究进展

\section{Progress in the Studies on Synthesis and Biological Properties of Dithiocarbamates}

\author{
Li, Ridong Wang, Yuanqiang Ge, Zemei Li, Runtao* \\ (State Key Laboratory of Natural and Biomimetic Drugs, School of Pharmaceutical Sciences, \\ Peking University, Beijing 100191)
}

\begin{abstract}
Due to the diverse biological activities of dithiocarbamates, their researches of synthesis and pharmacological activity have been attracting considerable interest in the areas of organic chemistry and medicinal chemistry. In the aspect of synthetic methods, this review is focused on the progress in the synthesis of dithiocarbamates under the following conditions: strong alkali, weak alkali, using water or ionic liquids as solvent and solvent-free conditions. In the aspect of biological activities, This review is focused on the progress in the following activities of dithiocarbamates: the treatment of chronic alcoholism, antioxidant, antiviral, antibacterial, cholinesterase inhibition, cancer prevention and antitumor activities.
\end{abstract}

Keywords dithiocarbamates; synthesis; biological activity; progress

氨基二硫代甲酸酯类化合物 $(\mathbf{1}$, 图 1)是一类重要的 含硫化合物, 具有广泛的生物活性, 主要表现为抗 菌 $^{[1 \sim 4]}$ 、抗病毒 ${ }^{[5]}$ 、抗氧化 ${ }^{[6 \sim 9]}$ 以及治疗慢性酒精中 毒 $^{[10]}$ 和重金属中毒 ${ }^{[11,12]}$ 等. 近年来, 越来越多的研究发 现, 氨基二硫代类化合物具有较好的肿瘤预防 ${ }^{[13]}$ 和抗 肿瘤活性 ${ }^{[14]}$. 鉴于氨基二硫代甲酸酯类化合物以其独 特的结构和性质在合成药物中占有重要的位置, 对含有 此类结构单元化合物的合成方法及生物性能的研究, 引 起了国内外学者的关注, 进行了广泛深入的探讨, 取得 了许多突破性的进展. 本文综述了近年来氨基二硫代甲 酸酯类化合物的合成及其生物活性研究进展.

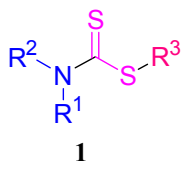

图 1 氨基二硫代甲酸酯类化合物结构通式

Figure 1 General structure of dithiocarbamates

\section{1 氨基二硫代甲酸酯类化合物的合成方法研究 进展}

氨基二硫代甲酸酯类化合物作为一类重要的含硫 化合物, 在有机化学和药物化学领域具有广泛的应用, 其合成方法的研究受到长期广泛的关注, 并且在最近十 几年取得了较大进展. 在早期, 氨基二硫代甲酸酯类化

\footnotetext{
*E-mail: lirt@bjmu.edu.cn

Received April 27, 2015; revised May 22, 2015; published online June 4, 2015.

Project supported by the National Natural Science Foundation of China (Nos. 21172011, 21372019).

国家自然科学基金(Nos. 21172011, 21372019)资助项目.
} 
合物通过胺与有毒且不易得的试剂, 如硫代光气 (thiophosgene $)^{[15]}$, 异硫氰酸酯(isothiocyanates) ${ }^{[16]}$ 等反应 制得. 近年来, 胺与二硫化碳和亲电试剂一锅法反应合 成氨基二硫代甲酸酯类化合物的方法受到越来越多的 关注和重视.下面主要对在强碱条件下、弱碱条件下、 以水为溶剂、以离子液为溶剂和无溶剂条件下合成氨基 二硫代甲酸酯类化合物的方法进行综述.

\section{1 强碱条件下的合成方法}

胺、二硫化碳和卤代烃在 $\mathrm{KOH}, \mathrm{NaOH}$ 或 $\mathrm{NaH}$ 等 强碱存在下反应 ${ }^{[17]}$, 是制备氨基二硫代甲酸酯类化合 物常用的方法. 通常是先由胺与二硫化碳在强碱性条件 下，在适当的溶剂中(常用乙醇作溶剂)形成对应的氨基 二硫代甲酸盐, 然后, 再与卤代烃反应形成氨基二硫代 甲酸酯类化合物(Scheme 1) $)^{[17]}$.

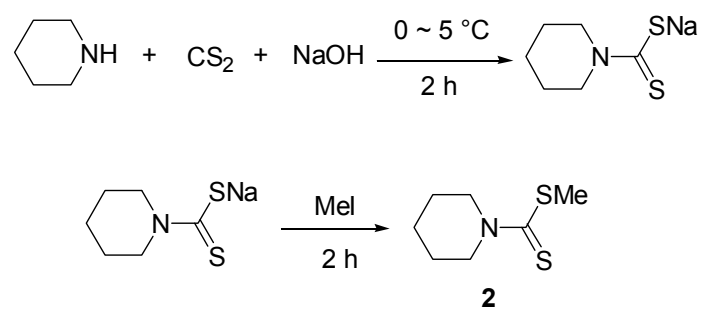

图式 1 强碱条件下制备氨基二硫代甲酸酯类化合物 Scheme 1 Synthesis of dithiocarbamates under strong alkali condition

该方法原料易得, 收率通常较高, 对于一些反应活 性较低的胺同样适用. 然而, 由于反应是在强碱性条件 下进行, 对于一些含有对碱敏感基团的胺和卤代烃则不 适用; 另外, 反应要分两步完成, 操作比较麻烦, 因而 限制了该方法的适用范围.

2013 年, Azizi 等 ${ }^{[18]}$ 报道了采用氢氧化钠作为催化 剂，催化芳香醛、芳香酮、脂肪胺和二硫化碳四组分反 应，以较好的收率得到相应的氨基二硫代甲酸酯化合物 (Eq. 1).

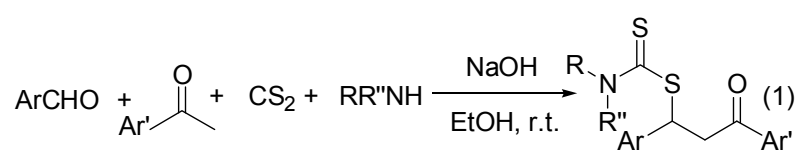

3

\section{2 弱碱条件下的合成方法}

\subsection{1 碳酸铯作用下的合成方法}

Jung 课题组 ${ }^{[19]}$ 研究发现, 在弱碱碳酸铯 $\left(\mathrm{Cs}_{2} \mathrm{CO}_{3}\right)$ 和 相转移催化剂四丁基碘化铵(TBAI)的作用下，脂肪胺、 芳香胺、二胺或氨基醇与二硫化碳和卤代烃反应可方便 地制得相应的氨基二硫代甲酸酯类衍生物(Scheme 2).

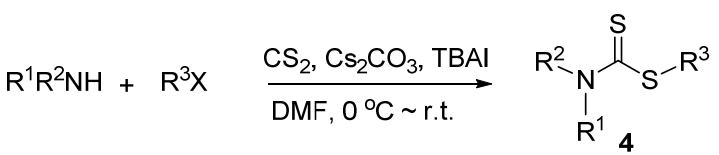

$$
\begin{aligned}
& \underset{\mathrm{H}}{\stackrel{\mathrm{N}}{\mathrm{N}_{n}} \mathrm{NH}_{2}+\mathrm{R}^{\prime} \mathrm{X}} \underset{\mathrm{DMF}, 0^{\circ} \mathrm{C} \sim \text { r.t. }}{\stackrel{\mathrm{CS}_{2}, \mathrm{Cs}_{2} \mathrm{CO}_{3} \text {, TBAI }}{\longrightarrow}}
\end{aligned}
$$

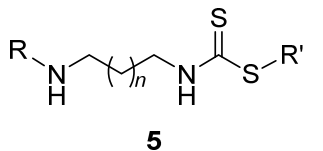

$$
\begin{aligned}
& \underset{\mathrm{HO}}{ } \mathrm{T}_{\mathrm{n}} \mathrm{NH}_{2}+\mathrm{R}^{\prime} \mathrm{X} \frac{\mathrm{CS}_{2}, \mathrm{Cs}_{2} \mathrm{CO}_{3}, \mathrm{TBAl}}{\mathrm{DMF}, 0^{\circ} \mathrm{C} \sim \text { r.t. }} \\
& { }_{\mathrm{HO}} \overbrace{\mathrm{H}}^{\mathrm{N}} \overbrace{\mathrm{S}^{-}}^{\mathrm{S}}
\end{aligned}
$$

图式 $2 \mathrm{Cs}_{2} \mathrm{CO}_{3}$ 作用下制备氨基二硫代甲酸酯类衍生物

Scheme 2 Synthesis of dithiocarbamates utilizing $\mathrm{Cs}_{2} \mathrm{CO}_{3}$

2004 年, Salvatore 课题组 ${ }^{[20]}$ 研究发现, 同样是在碳 酸铯 $\left(\mathrm{Cs}_{2} \mathrm{CO}_{3}\right)$ 和相转移催化剂四丁基碘化铵(TBAI)的作 用下，肼、二硫化碳和卤代烃在室温下反应，得到相应 的肼基二硫代甲酸酯(Eq. 2).

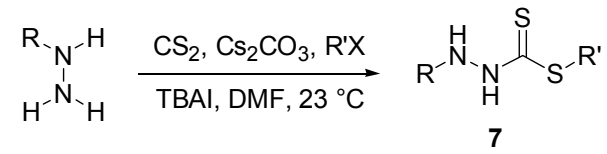

\subsection{2 无水磷酸钾作用下的合成方法}

我们课题组研究发现: 在无水磷酸钾存在下，以丙 酮或 DMF 为溶剂, 胺与二硫化碳和卤代烃三组分反应, 可以快速、高收率地制得氨基二硫代甲酸酯 (Eq. 3) ${ }^{[21 \mathrm{a} \sim 21 \mathrm{~d}]}$. 该方法具有收率高, 反应条件温和，操 作简便，催化剂常见、价格低廉等优点，尤其是适用于 含有活泼基团的胺和卤代烃，适用范围比较广泛.

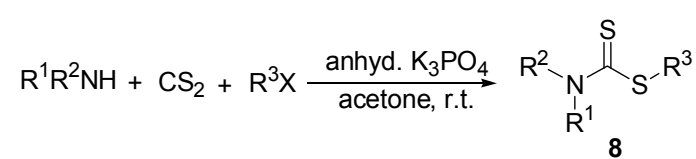

用环氧化合物代替卤代烃 ${ }^{[21 \mathrm{e}]}$ ，在该反应条件下， 反应也能够非常顺利地进行，且在该反应中可将羟基引 入目标产物中(Eq. 4).

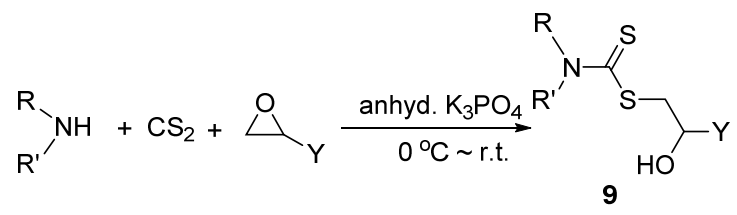

此外, 我们还将该方法扩展到胺与二硫化碳和 $\alpha, \beta$ 不饱和化合物的 Michael 加成反应中(Eq. 5 ${ }^{[21 f]}$ ，使无水 
磷酸钾的催化范围不仅局限于卤代烃、环氧化合物, 而 是使用面更广.

$$
\begin{aligned}
& \underset{\mathrm{R}^{\prime}}{\stackrel{\mathrm{R}}{\mathrm{R}^{\prime}} \mathrm{NH}}+\mathrm{CS}_{2}+\mathrm{XCH}=\mathrm{CH}-\mathrm{EWG} \underset{\mathrm{MeOH} \text {, r.t. }}{\stackrel{\text { anhyd. } \mathrm{K}_{3} \mathrm{PO}_{4}}{\longrightarrow}} \\
& \mathrm{R}^{\prime \prime}{ }_{\mathrm{N}}^{\mathrm{R}} \stackrel{\mathrm{C}}{\mathrm{S}} \text { EWG }
\end{aligned}
$$

10

作为该方法的延伸, 我们发现了一种无水磷酸钾催 化的四组分反应合成 3-烷基二硫代羰基啞唑烷衍生物 的新方法 ${ }^{[22]}$. 在无水磷酸钾的存在下, 氨基乙醇和酮形 成噁唑烷中间体, 不经分离, 直接与二硫化碳和卤代烃 反应以较高的收率得到相应的 3-烷基二硫代羰基噁唑 烷衍生物(Eq. 6).

$$
\begin{aligned}
& \mathrm{R}^{2} \stackrel{\mathrm{O}}{\mathrm{R}^{3}}+\underbrace{\mathrm{NH}_{2}}_{\mathrm{R}^{1}}+\mathrm{CS}_{2}+\mathrm{R}^{4} \mathrm{X} \underset{\text { r.t. }}{\stackrel{\text { anhyd. } \mathrm{K}_{3} \mathrm{PO}_{4}}{\longrightarrow}} \\
& \underbrace{R^{2}}_{N} \|_{S}^{S}-R^{4}
\end{aligned}
$$

11

\subsection{3 碱性氧化铝作用下的合成方法}

由于芳香胺的反应活性较低, 很少有文献报道以芳 香胺为底物通过 Michael 加成反应制备芳氨基二硫代甲 酸酯类化合物. 本课题组研究发现, 在固体介质碱性氧 化铝的催化下, 芳香胺、二硫化碳和缺电子烯烃通过 $S$-Michael 加成反应可非常容易得到芳氨基二硫代甲酸 酯类化合物 $(\mathrm{Eq} .7)^{[23]}$. 该方法操作简便, 收率高, 而且 固体介质碱性氧化铝可以回收套用.

$$
\mathrm{ArNH}_{2}+\mathrm{CS}_{2}+\mathrm{R}^{1} \curvearrowright \mathrm{R}^{2} \stackrel{\text { alkaline } \mathrm{Al}_{2} \mathrm{O}_{3}}{\longrightarrow} \mathrm{Ar}^{-} \prod_{\mathrm{S}}^{\mathrm{N}} \prod_{\mathrm{R}^{1}}^{\mathrm{S}} \mathrm{R}^{2}(7)
$$

\subsection{4 碳酸钾作用下的合成方法}

2013 年, 魏运洋课题组 ${ }^{[24]}$ 报道了碳酸钾作催化剂, 二氧六环为溶剂, $110{ }^{\circ} \mathrm{C}$ 的条件下, $N$-对甲苯磺酰腙与 二硫化碳和胺反应合成氨基二硫代甲酸酯类衍生物(Eq. 8). 其中 $N$-对甲苯磺酰腙可由芳香酮、醛或脂肪酮、醛 与对甲苯磺酰肼在反应体系中直接生成, 不用分离.

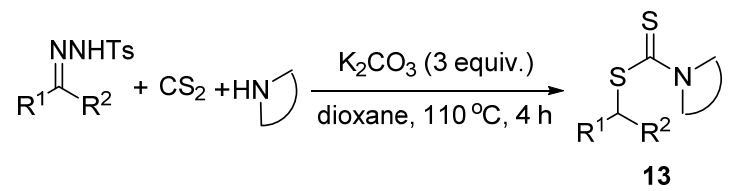

\subsection{5 三乙胺作用下的合成方法}

2009 年, Sureshbabu 等 ${ }^{[25]}$ 报道了在三乙胺的存在下,
氨基酸酯、二硫化碳和 N-保护的氨基碘化物反应，制备 含有氨基二硫代甲酸酯片段的拟肽类化合物(Eq. 9). 该 反应条件温和，不会引起产物手性中心的消旋.
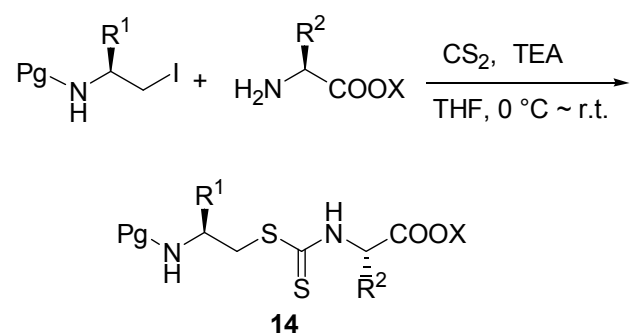

$\mathrm{Pg}=$ Fmoc, Boc; $\mathrm{X}=\mathrm{Me}, \mathrm{Et}$

\section{3 以水为溶剂的合成方法}

2006 年, Saidi 课题组 ${ }^{[26 a]}$ 首次报道了在没有碱性催 化剂的条件下, 以水为溶剂, 胺、二硫化碳与 $\alpha, \beta$-不饱 和化合物反应合成相应的氨基二硫代甲酸酯类化合物 (Scheme 3). 该反应避免了使用有毒的有机溶剂和催化 剂，反应条件温和，操作简便。当反应产物为固体时， 通过过滤就能得到纯的产物，避免了萃取、柱层析等后 处理操作. 此外, 该课题组将该方法扩展到胺、二硫化 碳和环氧化物 ${ }^{[26 b]}$ 或卤代烃 ${ }^{[26 c]}$ 的反应中(Scheme 3 ), 同 样取得很好的效果. 但是当底物为反应活性较低的芳香 胺时，该方法不适用。

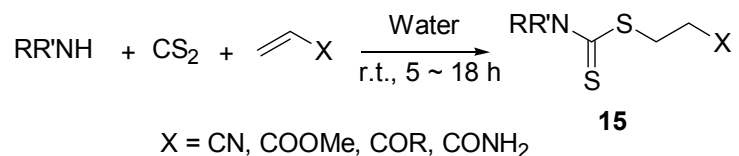
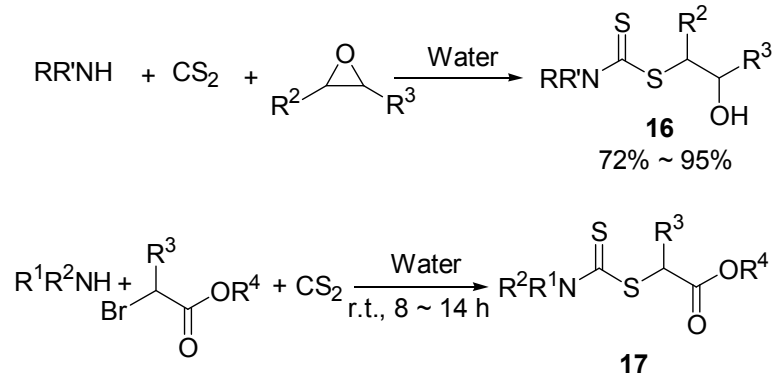

图式 3 以水为溶剂制备氨基二硫代甲酸酯类化合物

Scheme 3 Synthesis of dithiocarbamates in water

2008 年, Ranu 等 ${ }^{[27]}$ 报道了采用铜纳米粒作催化剂, 水作溶剂，回流条件由胺、二硫化碳和芳基碘或苯乙烯 基溴反应合成 $S$-芳基氨基二硫代甲酸酯或 $S$-乙烯基氨 基二硫代甲酸酯衍生物(Eq. 10). 该反应收率高(76\% 95\%), 对于制备 $S$-乙烯基氨基二硫代甲酸酯衍生物具 有很高的立体选择性，而且催化剂可以循环利用，环境 友好。 


$$
\mathrm{RX}+\mathrm{CS}_{2}+\mathrm{HN} \bigcirc \frac{\text { Cu nanoparticles }}{\mathrm{H}_{2} \mathrm{O} \text {, reflux }}+\mathrm{R}^{-\mathrm{S}_{\mathrm{S}}} \underset{\mathbf{1 8}}{ } \prod^{\mathrm{N} \backslash}
$$

2011 年, Ranu 等 ${ }^{[28]}$ 采用芳基重氮氟硣酸盐为底物, 以水为溶剂, 在室温条件下与胺和二硫化碳反应, 制备 相应的 $S$-芳基氨基二硫代甲酸酯类化合物(Eq. 11). 该 反应操作简便, 环境友好, 为合成 $S$-芳基氨基二硫代甲 酸酯类化合物提供了一种高效、便捷的方法.

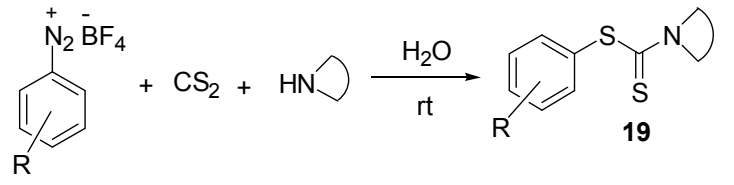

2009 年, Srivastava 等 $^{[29]}$ 报道了一种合成 $(E)$-和 $(Z)-$ 烯丙基氨基二硫代甲酸酯衍生物的新方法. 该方法采用 Baylis-Hillman 醋酸加合物为底物, 以水为溶剂, 在室温 条件下与胺和二硫化碳反应, 高收率( $80 \%$ ～94\%)地得 到相应的 $(E)$-和 $(Z)$ - 烯丙基氨基二硫代甲酸酯衍生物 (Eq. 12). 该反应避免了使用碱和有毒的有机溶剂, 绿色 环保, 而且反应产物在碱催化的条件下能够发生分子内 环合反应, 形成具有药理活性的 1,3-噻嗪类化合物.
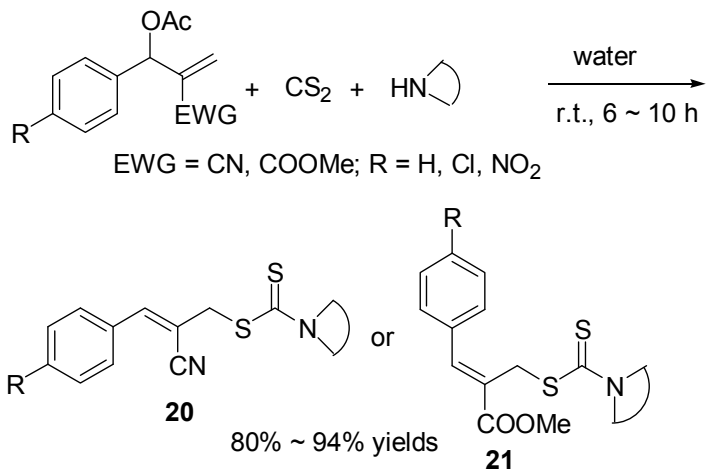

2010 年, Halimehjani 等 ${ }^{[30]}$ 报道了一种利用马氏加成 反应制备氨基二硫代甲酸酯类化合物的方法. 该反应以 水为溶剂, 胺和二硫化碳首先形成氨基二硫代甲酸, 然 后与烷基乙烯基醚发生马氏加成反应, 以完全的区域专 一性得到相应的马氏加成产物(Eq. 13). 该方法具有收 率高、无催化剂、反应条件温和等优点.

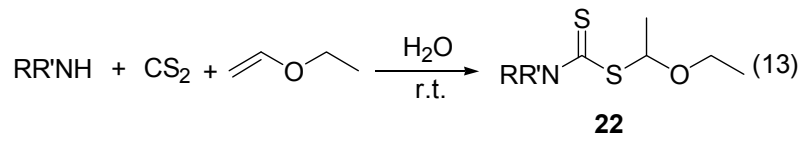

2011 年, Karmakar 课题组 ${ }^{[31]}$ 以水为溶剂, 采用 $\mathrm{MgO}$ 纳米晶体催化胺、二硫化碳与 $\alpha, \beta$-不饱和化合物反 应, 快速高效地合成相应的氨基二硫代甲酸酯衍生物 (Eq. 14).

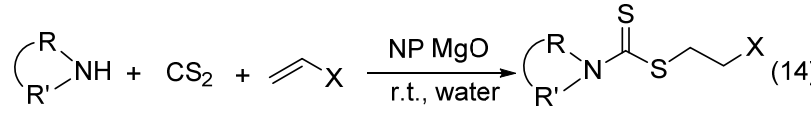

23

2012 年, Gholibeglo 等 ${ }^{[32]}$ 利用超声的方法, 可在水 相快速地制备氨基二硫代甲酸酯类化合物. 与常规方法 相比, 超声促进的反应仅需 $10 \mathrm{~min}$ 就能完成, 大大地缩 短了反应时间(Scheme 4)

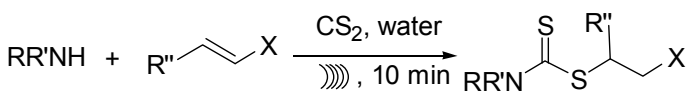

$$
\begin{aligned}
& \mathrm{X}=\text { COOMe, COMe, CN } 24
\end{aligned}
$$

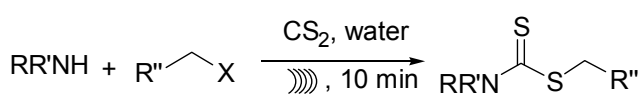

$$
\begin{aligned}
& \mathrm{X}=\mathrm{I}, \mathrm{Br}, \mathrm{Cl}
\end{aligned}
$$

图式 4 超声促进合成氨基二硫代甲酸酯衍生物 Scheme 4 Synthesis of dithiocarbamates under ultrasound irradiation

\section{4 以离子液为溶剂的合成方法}

2008 年, Ranu 课题组 ${ }^{[33]}$ 首次报道了在离子液 $[\mathrm{pmIm}] \mathrm{Br}$ 中合成氨基二硫代甲酸酯类化合物的新方法 (Scheme 5). 在该方法中, 离子液作为溶剂和催化剂, 适用范围广, 能够在温和的条件下促进反应的快速进行 $(10 \sim 30 \mathrm{~min})$, 而且离子液可以回收再利用, 对环境友 好.

\section{5 无溶剂条件下的合成方法}

2006 年, Saidi 等 ${ }^{[34 a]}$ 首次报道了在无溶剂、无催化 剂的条件下, 胺、二硫化碳与卤代烃反应合成相应的氨 基二硫代甲酸酯类化合物. 该反应原子经济性好, 操作 简单，条件温和，绿色环保，且适用于放大生产. 此外, 他们对该方法的适用范围进行了扩展. 研究发现，该方 法同样适用于环氧化物 ${ }^{[34 \mathrm{~b}]} 、 \alpha, \beta$-不饱和化合物 ${ }^{[34 \mathrm{c}]}$ 或炔 烃 ${ }^{[34 d]}$ 与胺和二硫化碳的反应(Scheme 6).

2012 年, Misra 课题组 ${ }^{[35]}$ 报道了无溶剂、无催化剂 的条件下，糖基溴化物、胺和二硫化碳三组分在室温下 发生反应，快速、高收率地得到相应的 $S$-糖基氨基二硫 发生反应，快速、高收率地得到相应的 $S$-糖基氨基二硫 代甲酸酯衍生物(Eq. 15).

2014 年, Singh 等 ${ }^{[36]}$ 报道了无溶剂的条件下，甲基 芳烃经 TBHP/NBS 处理后与胺和二硫化碳反应, 得到相 应的氨基二硫代甲酸酯类化合物(Eq. 16). 该方法提供 了一种由简单易得的甲基芳烃直接制备氨基二硫代甲 酸酯类化合物的新途径. 


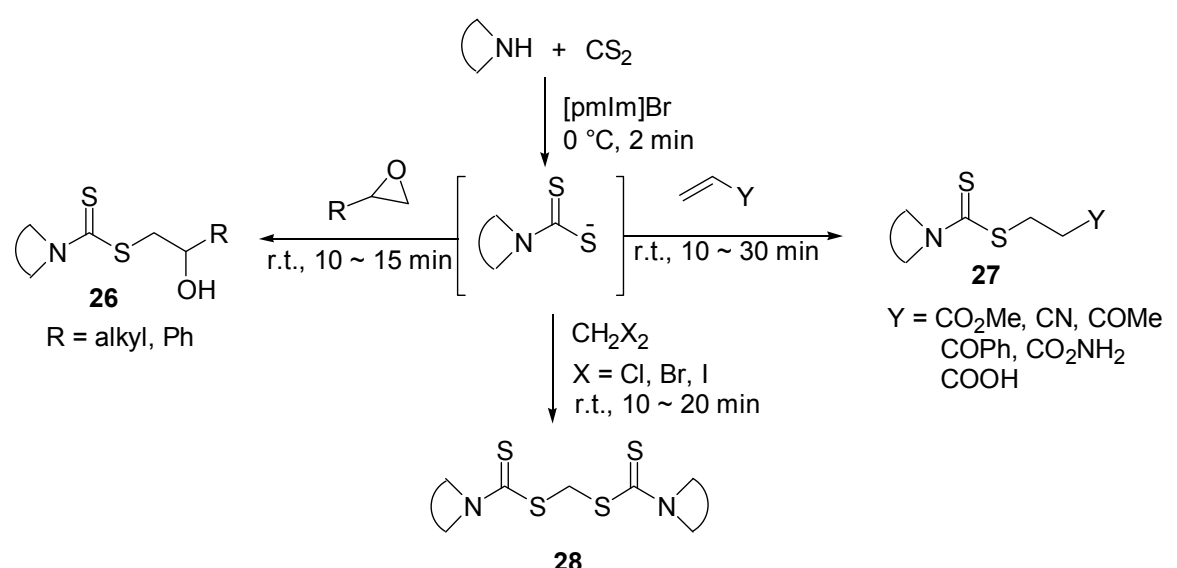

图式 5 以离子液为溶剂合成氨基二硫代甲酸酯类化合物

Scheme 5 Synthesis of dithiocarbamates using ionic liquids as solvent

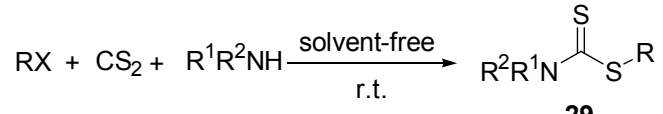

$$
\begin{aligned}
& 29
\end{aligned}
$$

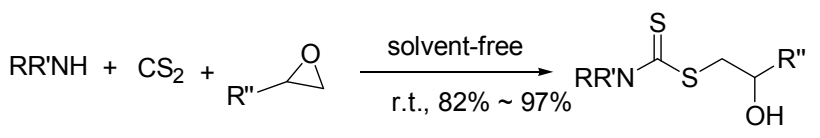

$$
\begin{aligned}
& 30 \\
& \mathrm{RR}^{\prime} \mathrm{NH}+\mathrm{R}_{3} \leadsto \mathrm{X}+\mathrm{CS}_{2} \underset{\text { r.t., } 80 \% \sim 97 \%}{\stackrel{\text { solvent-free }}{\longrightarrow}} \mathrm{RR}^{\prime} \mathrm{N}_{\mathrm{S}} \\
& \mathrm{X}=\mathrm{COOMe}, \mathrm{COPh}, \mathrm{COMe}, \mathrm{CONH}_{2}, \mathrm{CN} \\
& 31 \\
& \mathrm{RR}^{\prime} \mathrm{NH}+\mathrm{CS}_{2}+\mathrm{R}^{3}=\frac{\text { solvent-free }}{80^{\circ} \mathrm{C}} \mathrm{RR}^{\prime} \mathrm{H}_{\mathrm{R}^{3}}
\end{aligned}
$$

图式 6 无溶剂条件下制备氨基二硫代甲酸酯类化合物

Scheme 6 Synthesis of dithiocarbamates under solvent-free conditions

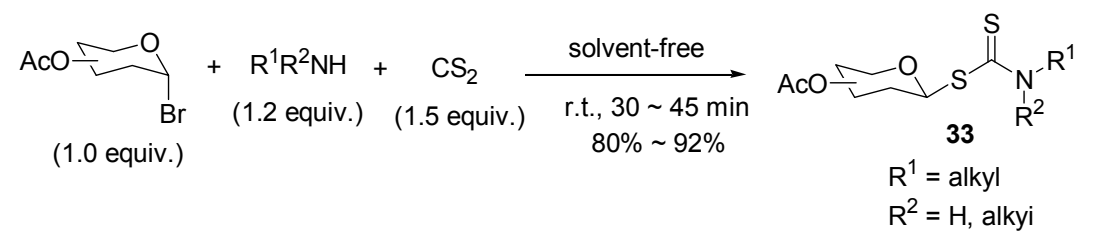

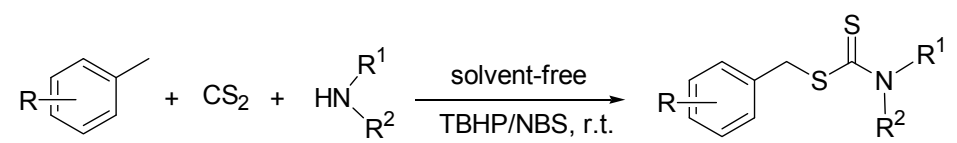

$$
\begin{aligned}
& 34
\end{aligned}
$$

\section{6 其他的合成方法}

2006 年, Chaturvedi 等 ${ }^{[37]}$ 报道了一种在 Mitsunobu 试剂( $\left.\mathrm{DEAD} / \mathrm{Ph}_{3} \mathrm{P}\right)$ 的存在下, 由醇、胺和二硫化碳反应
制备氨基二硫代甲酸酯衍生物的方法(Eq. 17). 该方法 条件温和, 化学选择性好, 提供了一种以醇为原料制备 氨基二硫代甲酸酯类化合物的新途径. 


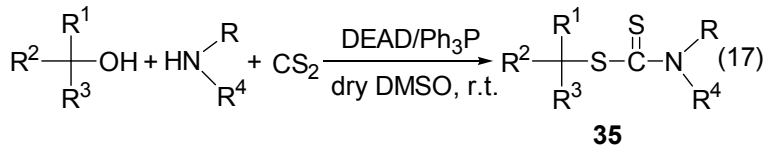

2012 年, Varma 课题组 ${ }^{[38]}$ 报道了一种模块化合成含 有氨基二硫代甲酸酯侧链的非天然 $\alpha$-氨基酸的方法. 在 DMF 为溶剂的条件下, 胺、二硫化碳和环状磺酰胺在室 温下反应, 可得到含有氨基二硫代甲酸酯侧链的非天然 $\alpha$-氨基酸(Scheme 7). 该方法操作简便, 收率高, 而且具 有很好的区域选择性和立体选择性.

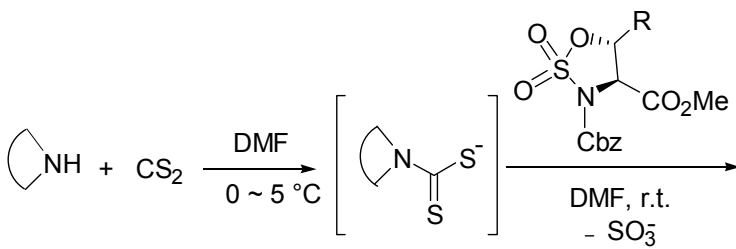

$$
\begin{aligned}
& \mathrm{C}_{\mathrm{S}}^{\mathrm{S}} \overbrace{\mathrm{NHCbz}}^{\mathrm{O}} \\
& 36
\end{aligned}
$$

图式 7 合成含有氨基二硫代甲酸酯侧链的非天然 $\alpha$-氨基酸 Scheme 7 Synthesis of dithiocarbamate pendant unnatural $\alpha$-amino acids

2015 年, Banerjee 等 ${ }^{[39]}$ 报道用介孔铜材料作催化剂, 在室温条件下催化芳香胺、二硫化碳和 $\alpha, \beta$-不饱和化合 物反应，以较高的收率得到 $N$-芳氨基二硫代甲酸酯类 化合物(Eq. 18).

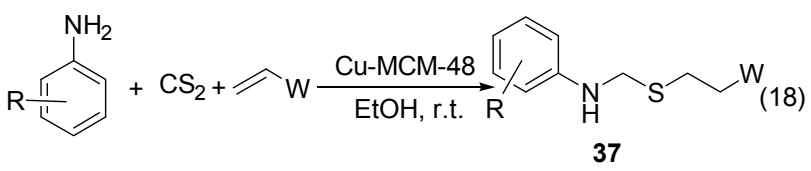

\section{2 氨基二硫代甲酸酯类化合物的生物活性研究 进展}

\section{1 治疗慢性酒精中毒}

双硫仑 ${ }^{[40,41]}$ (Disulfiram, DSF, 38, 图 2) 作为不可逆 的醛基脱氢酶抑制剂, 被批准用于治疗酒精中毒已有近 50 年的历史.

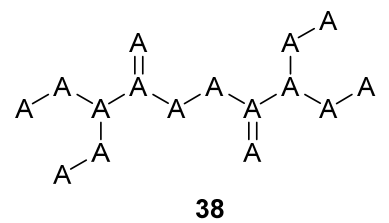

图 2 DSF 的结构

Figure 2 Structure of DSF

\section{2 抗氧化活性}

近年来发现氨基二硫代甲酸酯类化合物在细胞及 分子生物学上可作为抗氧化剂抑制脂质体和肝微粒体 中类脂的过氧化 ${ }^{[42 ~ 48]}$. 如吡咯烷二硫代甲酸(PDTC, 39, 图 3) 可抑制 NF- $\mathrm{kB}$ 的氧化活性 ${ }^{[47 \sim 9]}$. NF- $\mathrm{kB}$ 是一个转录 因子, 在免疫功能方面、细胞的生长与调亡中都起着重 要的作用. PDTC 还可以抑制胸腺细胞的坏死 ${ }^{[50]}$, 也可 以抑制白血病细胞 HL-60 增殖，诱导细胞凋亡 ${ }^{[51]}$.

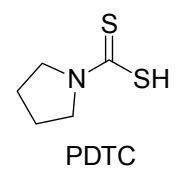

39

图 $3 \mathrm{PDTC}$ 的结构

Figure 3 Structure of PDTC

\section{3 抗病毒活性}

人鼻病毒(HRVs) 是导致感冒的主要原因, 因其易 发性以及缺乏有效的药物而使得研究新型的抗病毒药 物显得非常重要. 有研究表明, PDTC 具有非常有效的 抗病毒作用, 而且对几种细胞系的 HRVs 亚型都显示了 很好的抑制作用 ${ }^{[52]}$.

\section{4 抗菌活性}

$\beta$-内酰胺类抗生素类抗生素具有杀菌活性强、毒性 低、适应症广及临床疗效好等优点. Ohtake 课题组 ${ }^{[53]}$ 用 氨基二硫代甲酸酯片段替换 $\beta$-内酰胺类抗生素 $40 \mathrm{C}-2$ 位的噻唑基团，设计并合成了一系列含有 $\beta$-内酰胺结构 的氨基二硫代甲酸酯类化合物 41 (Scheme 8), 并评价该 类化合物的抗菌活性. 结果表明, 该类化合物均具有很 好的抗菌活性, 其中化合物 41a 和 41b 的活性最好, 对 金黄色葡萄球菌和上皮葡萄球菌的活性明显优于万古 霉素. 2001 年, 该课题组 ${ }^{[54]}$ 对化合物 $\mathbf{4 1}$ 的结构进行了改 造, 将双季铵化 1,4-二氮杂双环辛烷基团引入到 C-2 位 的氨基二硫代甲酸酯的骨架中，合成了一系列新结构类 型的抗菌剂. 研究发现, 化合物 $\mathbf{4 2}$ 显示出很好的抗耐甲 氧西林金黄色葡萄球菌的活性.

2009 年, Takii 等 ${ }^{[55]}$ 将氨基葡萄糖、氨基甘露糖和氨 基半乳糖与氨基二硫代甲酸酯基团进行拼合，设计并合 成了一系列含糖环骨架的氨基二硫代甲酸酯类化合物 43 46(图 4), 通过对该系列化合物的抗结核分支杆菌 活性的测试发现, 化合物 43 具有很好的抑菌活性. 构效 关系分析结果表明，在葡萄糖苷的 C-1 位引入氨基二硫 代甲酸酯基团对抗菌活性的提高起到了非常关键的作 用. 
<smiles>[R]c1nc(SC2=C(C(=O)O)N3C(=O)C(C(C)O)[C@H]3C2C)sc1[R]</smiles>

40<smiles>[R]N([R])C(=S)SC1=C(C(=O)O)N2C(=O)C(C(C)O)[C@H]2C1C</smiles>

41<smiles>C=CC(C)C1C(=O)N2C(=O)C(SC(=S)N3CC=C(C[N+]45CCC(CC4)N(C(=O)[O-])CC5)CC3)=C(C(N)O)C12</smiles>

42<smiles>CC(O)C1C(=O)N2C(C(=O)O)=C(SC(=S)N(C)CCO)C(C)C12</smiles>

$41 a$<smiles>CC(O)C1C(=O)N2C(C(=O)O)=C(SC(=S)N3CCCC(O)CC3)C(C)C12</smiles>

41b

图式 4 含氨基二硫代甲酸酯基团的 $\beta$-内酰胺类抗菌剂

Scheme 4 Novel dithiocarbamate carbapenemsl with anti-MRSA activity

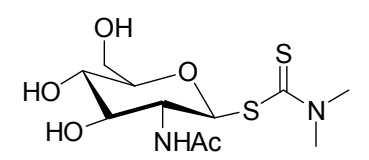

43

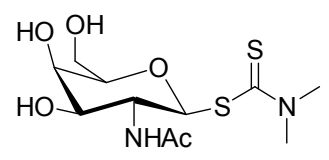

45

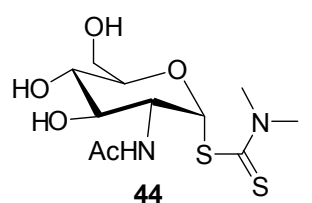

$\mathrm{S}$

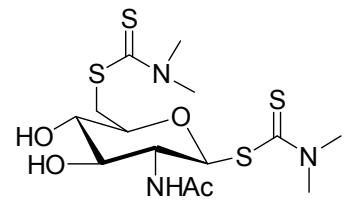

46
图 4 含糖环骨架的氨基二硫代甲酸酯类化合物

Figure 4 Dithiocarbamates containing sugar skeleton

酰化高丝氨酸内酯类(AHLs)化合物是革兰氏阳性 菌群体感应系统中最重要的一类信号分子. 2013 年, 刘 宏民等 ${ }^{[56]}$ 设计并合成了两个系列含高丝氨酸内酯结构 的氨基二硫代甲酸酯类化合物 47, 48(图 5). 抗菌活性结 果显示, 含取代 $N$-苯基酰胺结构的化合物 47 对革兰氏 阳性菌有较好的抑制活性, 其中当 $\mathrm{R}$ 为 $3-\mathrm{CF}_{3}$ 时活性最 好, 其对枯草芽孢杆菌的 $\mathrm{MIC}_{50}$ 达到 $1.443 \mu \mathrm{g} / \mathrm{mL}$. 而含 有疏水烷基链的化合物 48 并没有明显的抗菌活性.<smiles>[R]c1cccc(NC(=O)CSC(=S)N[C@@H]2CCOC2=O)c1</smiles>

47<smiles>CSC(=S)NC1CCOC1=O</smiles>

48
图 5 含氨基二硫代甲酸酯基团的 AHL 类似物

Figure 5 AHL analogues containing dithiocarbamate

2014 年, 吴秋叶等 ${ }^{[57]}$ 基于临床上应用的康唑类抗 真菌药物的结构特点, 在康唑类药物的分子骨架中引入 氨基二硫代甲酸酯基团, 设计并合成了一系列含有三氮
唑结构的氨基二硫代甲酸酯类化合物 49 (Eq. 18). 体外 抗真菌活性结果表明, 在分子结构中引入合适的氨基二 硫代甲酸酯侧链有利于提高化合物对念珠菌的抑制活 性. 通过考察氨基二硫代甲酸酯的氨基部分对活性的影 响发现，仲胺的抗真菌活性优于伯胺. 当氨基为不同苯 基取代的哌嗪基时，抗真菌活性最好，大部分化合物的 活性优于上市药物氟康唑和酮康唑.

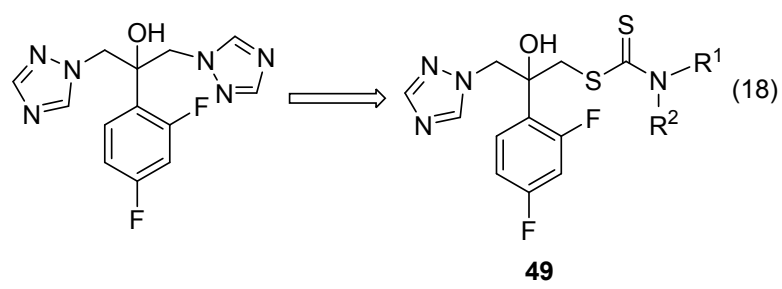

$R^{1} R^{2} \mathrm{NH}=$ primary amines, secondary amines

2015 年, Sharma 等 ${ }^{[58]}$ 将硫脲和氨基二硫代甲酸酯 基团拼合在一起，设计、合成了一系列含硫脲结构的氨 基二硫代甲酸酯类化合物 50(图 6), 并测试该系列化合 物对毛滴虫和精子的杀伤作用, 以及对逆转录酶的抑制 作用. 结果表明, 当 $R^{1}$ 为苯甲酰基, $R^{2}$ 为辛基时活性最 好 50a(图 6).

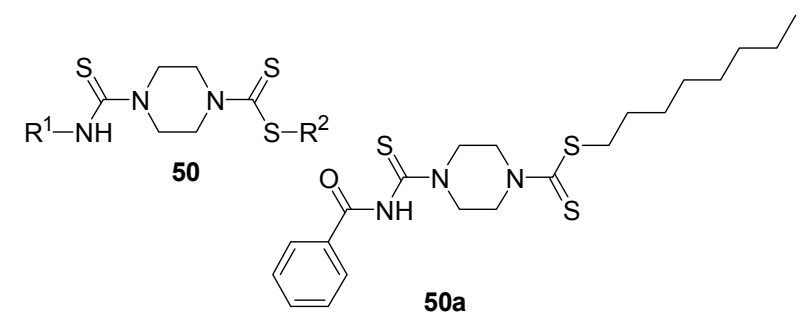

图 6 化合物 $\mathbf{5 0}$ 的结构

Figure 6 Structure of compound $\mathbf{5 0}$ 


\section{5 胆碱酯酶抑制活性}

2013 年, Altintop 等 ${ }^{[59 a]}$ 报道了一系列含吡唑啉结构 的氨基二硫代甲酸酯类化合物的合成及抗胆碱酯酶活 性研究. 发现化合物 51(图 7)具有抑制乙酰胆碱酯酶和 丁酰胆碱酯酶的活性. 其中化合物 51a 的活性最好, 对 乙酰胆碱酯酶和丁酰胆碱酯酶的 $\mathrm{IC}_{50}$ 分别为 $(0.72 \pm$ $0.06)$ 和 $(7.46 \pm 0.83) \mu \mathrm{g} / \mathrm{mL}$.

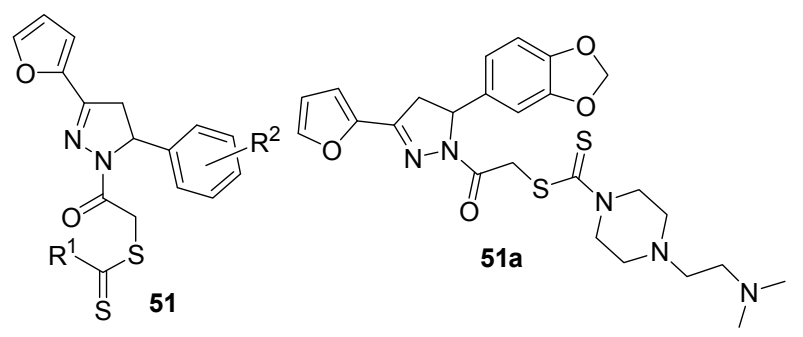

图 7 化合物 $\mathbf{5 1}$ 的结构

Figure 7 Structure of compound $\mathbf{5 1}$

2013 年, Altintop 课题组 ${ }^{[59 b]}$ 报道了一系列作为胆碱 酯酶抑制剂的氨基二硫代甲酸酯类化合物 52(图 8). 活 性结果表明, 当氨基为 4-甲基哌嗪(52a)和 4-乙基哌嗪 (52b)时, 化合物对乙酰胆碱酯酶和丁酰胆碱酯酶的抑 制活性最好.

\section{6 肿瘤预防作用}

Brassinin 是从十字花科植物卷心菜中提取出来的<smiles>[R16]N([R])C(=S)SCc1ccc(C(F)(F)F)cc1</smiles>

图 8 化合物 $\mathbf{5 2}$ 的结构

Figure 8 Structure of compound $\mathbf{5 2}$

一类具有吲哚环的色氨酸衍生物, 但由于其在天然产物 中含量很低, 且不易提取, 因此, 美国科学家运用化学 合成的方法制备了一系列 Brassinin 的衍生物, 并研究了 该系列化合物对肿瘤的预防作用(图 9 ( $^{[60 ~ 62]}$. 研究结果 表明, Brassinin 和 Cyclobrassinin 能够非常有效地阻止乳 腺癌和白血病的早期形成，被认为是一种有效的肿瘤预 防化合物.

Malachowski 课题组 ${ }^{[63]}$ 在篎选含有吲哚骨架的化合 物作为吲哚胺 2,3-双加氧酶(IDO)抑制剂的过程中发现, 天然产物 Brassinin 对 IDO 具有中等的抑制活性. 该课题 组合成了一系列 Brassinin 类似物, 测试了该系列化合物 对 IDO 的抑制活性. 构效关系研究结果表明，氨基二硫 代甲酸酯基团对抑制活性至关重要，吲哚环并不是产生 抑制活性所必需的母核, Brassinin 的 $S$-甲基部分被一些 大体积的芳香基团取代能显著提高抑制活性.<smiles>CC(C)=S=CCc1c[nH]c2ccccc12</smiles><smiles></smiles>

$\mathrm{N}$-Methoxylbrassinin<smiles>CC1=NCCC2(C=Nc3ccccc32)S1</smiles>

Spirohomobrassinin<smiles>CC(=S)NCc1c[nH]c2cccc(C)c12</smiles><smiles>CC(=S)NCc1c[nH]c2ccc(I)cc12</smiles>

5-Chlorobrassinin<smiles>CC(C)=NCc1c(C)sc(C)c1C(C)C</smiles><smiles>CC(C)=CCc1c[nH]c2c(C)cccc12</smiles>

7-Methylbrassinin<smiles>CSC1=NCC2(C1)C(=O)Nc1ccccc12</smiles>

图 9 Brassinin 及其衍生物的结构

Figure 9 Structures of brassinin and its derivatives 
菜菔硫烷(Sulforaphane, 53, 图 10)是 Posner 等从十 字花科植物中提取出的一种脂肪族异硫氰酸酯化合物, 研究发现该化合物具有很好的肿瘤预防作用, 它不直接 与自由基或活性氧物质发生反应, 而是通过激活 II 相代 谢酶基因上游启动子区域一些共同的抗氧化反应元件, 诱导 II 相代谢酶基因的表达, 以增强机体抗氧化、抗突 变和抗肿瘤的能力 ${ }^{[64,65]}$.

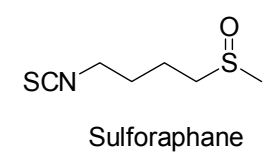

53

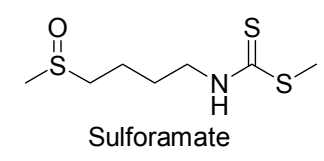

54<smiles>CSC(=S)NCCCCC(C)=O</smiles>

图 10 Sulforaphane、sulforamate 和 oxomate 的结构 Figure 10 Structures of sulforaphane, sulforamate and oxomate

基于 Sulforaphane 和 Brassinin 的肿瘤预防活性结 果, Pezzuto 等 ${ }^{[66]}$ 利用拼合原理将 Sulforaphane 分子中的 甲基亚砜基团和 Brassinin 分子中的氨基二硫代酯基同 时引入一个化合物中, 设计合成了新化合物 Sulforamate 54(图 10), 并对该化合物进行了肝肿瘤细胞 Hepalclc 7 的醌氧化还原酶 $(\mathrm{QR})$ 诱导活性试验. 结果发现, Sulforamate 与 Sulforaphane 的诱导活性相当, 但毒性却降 低了三分之二. 药理学研究表明, 该类化合物是通过激 活 II 相代谢酶从而达到肿瘤化学预防的作用. 在上述研 究结果的基础上, Pezzuto 等 ${ }^{[67]}$ 通过对 Sulforamate 进一 步的结构优化得到了化合物 Oxomate 55(图 10), 该化合 物同样具有很好的肿瘤预防作用.

\section{7 抗肿瘤活性}

Supuran 研究组 ${ }^{[68,69]}$ 一直致力于碳酸䣶酶抑制剂方 面的研究. 他们将磺酰胺结构与氨基二硫代甲酸酯结构 连接起来, 设计了一系列新型的氨基二硫代甲酸酯类化 合物 56(图 11), 考察其抗肿瘤活性, 并以此为探针, 研 究其对碳酸酩酶的抑制活性及该类化合物的抗肿瘤机 制.

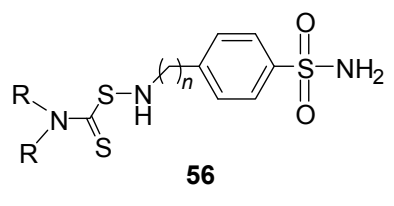

图 11 化合物 56 的结构

Figure 11 Structure of compound 56

初步活性结果显示, 当 $\mathrm{R}$ 为甲基、乙基, $n$ 为 $1 \sim 3$
时，表现出了很好的生物活性; 对于不同的肿瘤细胞株, 半数生长抑制率 $\left(\mathrm{GI}_{50}\right)$ 为 $10 \sim 65 \mathrm{nmol} / \mathrm{L}$, 对 $\mathrm{hCAII}$ 的结 合常数 $\left(K_{\mathrm{i}}\right)$ 为 $10 \sim 35 \mathrm{nmol} / \mathrm{L}$.

Özlen 研究组 ${ }^{[70]}$ 将内磺酰胺结构与氨基二硫代甲酸 酯结构结合, 设计合成了一系列含内磺酰胺结构的氨基 二硫代甲酸酯类化合物 57(图 12), 期望发现既有抗菌活 性又有抗肿瘤活性化合物.

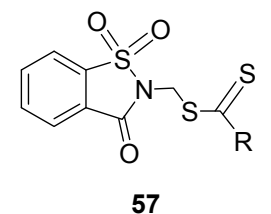

图 12 化合物 $\mathbf{5 7}$ 的结构

Figure 12 Structure of compound 57

初步活性结果显示, 当 $\mathrm{R}$ 为二乙氨基、哌啶基、甲 基哌嗪基时, 表现出了很好的抗肿瘤活性. 然而, 该课 题组没有进一步总结其构效关系.

Zahran 研究组 ${ }^{[71]}$ 在沙利度胺的结构中引入氨基二 硫代甲酸酯基团, 设计合成了一系列含有沙利度胺母核 的氨基二硫代甲酸酯类化合物，考察了该系列化合物在 体外对艾氏腹水瘤细胞和在体内对艾氏腹水瘤的抑制 作用, 并研究了它们的抗肿瘤机制.

活性结果显示, 对于化合物 58(图 13), 当氨基为 $N$-(2-氨基乙基)哌啶 (58a) 和 1-氨基-4-甲基哌嗪(58b)时, 具有非常好的抗肿瘤活性，而且没有肝脏毒性. 该系列 化合物的抗氧化、清除自由基的作用可能是其发挥抗肿 瘤作用的机制之一。<smiles>CCCCC(=O)SCC1C(=O)CCC2N3C(=O)C(CCC12C(=O)O)C(=O)c1ccccc13</smiles>

58

\section{$58 a$}<smiles>CCCCCCCCCCCCCCCC1C(=O)CCC(N2C(=O)c3ccccc3C2=O)C1=O</smiles>

$58 b$

图 13 化合物 58 的结构

Figure 13 Structure of compound 58

黄酮及其衍生物具有广泛的生物学活性, 杨光富 等 ${ }^{[72]}$ 将氨基二硫代甲酸酯基团引入到黄酮的结构中, 设计、合成了 4 个系列含有黄酮骨架的氨基二硫代甲酸 酯类化合物 59 62(图 14), 并评价它们对多种肿瘤细胞 
的体外抑制活性试验. 结果显示, 对于化合物 59, 当 $\mathrm{X}=\mathrm{Cl}, \mathrm{R}=\mathrm{H}$, 氨基部分为哌啶时, 活性最好; 对于化 合物 60, 当 $\mathrm{R}=6-\mathrm{Cl}$, 氨基部分为哌啶时, 活性最好. 其 抗肿瘤作用机制与阻断细胞周期和诱导细胞调亡有关.<smiles>[X]c1c(SC(=S)N([R])[R])oc2cccc([R])c2c1=O</smiles>

59<smiles>[R]c1ccc2occ(CSC(=S)N([R])[R])c(=O)c2c1</smiles>

61<smiles>[R]c1cccc2occ(CSC(=S)N([R])[R])c(=O)c12</smiles>

60
图 14 化合物 59 62 的结构

Figure 14 Structures of compound $59 \sim 62$

查尔酮是天然产物中的重要药效团, 也是合成黄酤 和异黄酮类化合物的重要前体, 具有广泛的生物学活 性. 朱海亮等 ${ }^{[73]}$ 将氨基二硫代甲酸酯基团引入到查尔 酮的分子骨架中, 设计、合成了一系列含有查尔酮分子 骨架的氨基二硫代甲酸酯类化合物 63(图 15), 并对所合 成的化合物进行抗增殖和抗微管蛋白聚合的活性测试. 活性结果表明, 化合物 63a 表现出了良好的活性, 其对 $\mathrm{MCF}-7$ 细胞生长的 $\mathrm{IC}_{50}$ 为 $0.04 \mu \mathrm{mol} / \mathrm{L}$, 抑制微管蛋白聚 合的 $\mathrm{IC}_{50}$ 为 $6.8 \mu \mathrm{mol} / \mathrm{L}$, 该化合物可能是一类新型的微 管蛋白聚合抑制剂.<smiles>[R]c1ccc(C(=O)CC(SC(=S)N(CC)CC)c2ccc([R])c([R])c2[R])cc1[R]</smiles>

63

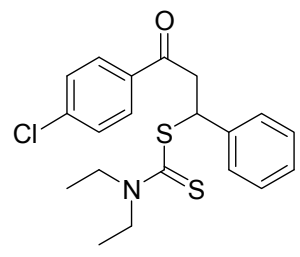

$63 a$
图 15 化合物 63 的结构

Figure 15 Structure of compound 63

Manga 课题组 ${ }^{[74]}$ 将氨基二硫代甲酸酯基团分别引 入到苯并咪唑和查尔酮的分子骨架中, 设计、合成了两 个系列的氨基二硫代甲酸酯类化合物 64 和 65(图 16), 并评价了这两类化合物的抗有丝分裂能力. 对于化合物 64, 当氨基为哌啶时活性最好; 对于化合物 65 , 当氨基 为 2-氨基苯并噻唑时活性最好.

Sharma 研究组 ${ }^{[75]}$ 将烷基磷酸酯基团和氨基二硫代 甲酸酯基团拼合在一起, 设计、合成了一系列含磷酸酯 基团的氨基二硫代甲酸酯类化合物 66(图 17), 并测试了

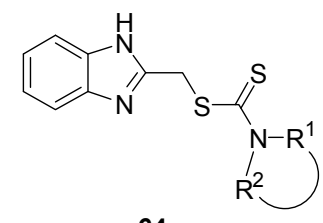

64<smiles>[R]N1CCCCCC1=S</smiles>

65
图 16 化合物 64 和 65 的结构

Figure 16 Structures of compounds 64 and 65

该系列化合物对与激素相关的肿瘤细胞增殖的抑制作 用. 当 $R^{1} R^{2} N$ 为哌啶基, $R^{3}$ 为十六烷基时(66a)对前列腺 癌细胞 DU-145 增殖的抑制活性最好, $\mathrm{IC}_{50}$ 为 $14.69 \pm 1.0$ $\mu \mathrm{mol} / \mathrm{L}$. 当 $\mathrm{R}^{1} \mathrm{R}^{2} \mathrm{~N}$ 为哌啶基, $\mathrm{R}^{3}$ 为癸基时(66b)对乳腺癌 细胞 $\mathrm{MCF}-7$ 增殖的抑制活性最好, $\mathrm{IC}_{50}$ 为 $7.24 \pm 2.15$ $\mu \mathrm{mol} / \mathrm{L}$. 作用机制研究结果表明, 化合物 $66 \mathbf{b}$ 能显著下 调磷酸化的 Akt 在乳腺癌细胞中的表达. 他们推测该类 化合物有可能是通过阻滞 Akt 通路抑制肿瘤增殖.

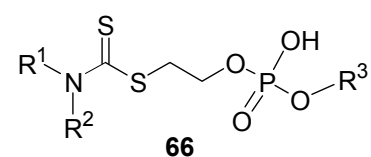

66a: $R^{1} R^{2} \mathrm{~N}=$ piperdinyl, $\mathrm{R}^{3}=\mathrm{CH}_{2}\left(\mathrm{CH}_{2}\right)_{14} \mathrm{CH}_{3}$

66b: $R^{1} R^{2} \mathrm{~N}=$ piperdinyl, $\mathrm{R}^{3}=\mathrm{CH}_{2}\left(\mathrm{CH}_{2}\right)_{8} \mathrm{CH}_{3}$

图 17 化合物 66 的结构

Figure 17 Structure of compound $\mathbf{6 6}$

曹胜利等 ${ }^{[76 ~ 78]}$ 将氨基二硫代甲酸酯结构片段引入 到 4(3H)-喹唑啉酮的 C-6 位，合成了一系列含有氨基二 硫代甲酸酯侧链的 4(3H)-喹唑啉酮衍生物 67, 68(图 18). 体外活性试验结果表明，对于化合物 67, 当氨基部分为 1-(4-氟苯基)哌嗪时, 其对人慢性髓性白血病 K562 细胞 的抑制活性最好, $\mathrm{IC}_{50}$ 为 $0.5 \mu \mathrm{mol} / \mathrm{L}$. 对于化合物 68, 当 $\mathrm{R}$ 为 3-氰基-3,3-二苯基丙基时, 其对乳腺癌细胞 MCF-7 的抑制活性最好, $\mathrm{IC}_{50}$ 为 $3.65 \mu \mathrm{mol} / \mathrm{L}$; 当 $\mathrm{R}$ 为 4-氟苄基 时, 其对人肺癌细胞 $\mathrm{A} 549$ 的抑制活性最好, $\mathrm{IC}_{50}$ 为 5.09 $\mu \mathrm{mol} / \mathrm{L}$.<smiles>[R]SC(=S)NCc1ccc2nc(C)[nH]c(=O)c2c1</smiles>

图 18 化合物 67 和 68 的结构

Figure 18 Structures of compounds 67 and 68

2013 年，曹胜利等 ${ }^{[79]}$ 对化合物 67 的结构进行改造, 用 2,4-二氨基喹唑啉替代化合物 67 中的 2-甲基-4(3H)喹唑啉酮结构单元，设计、合成了一系列 4-取代哌嗪-1二硫代甲酸酯衍生物 69(图 19), 并评价了该系列化合物 
对人肺癌(A549)、乳腺癌(MCF-7)、宫颈癌(Hela)和结肠 癌(HT29、HCT116)细胞增殖的抑制活性. 结果表明, 该 系列化合物对上述 5 种细胞系表现出广谱的抗增殖活性 $\left(\mathrm{IC}_{50}: 1.47 \sim 11.83 \mu \mathrm{mol} / \mathrm{L}\right.$ ), 尤其是当 $\mathrm{R}$ 为对甲氧基苯基 (69a)、对氟苯基 $(69 b)$ 和对硝基苯基 (69c) 时活性最好.<smiles>[R]N1CCN(C(=S)SCC2=CC3C(N)=NC(N)=NC3C=C2)CC1</smiles>

69

\section{9a: $\mathrm{R}=4-\mathrm{CH}_{3} \mathrm{OC}_{6} \mathrm{H}_{4}$ 69b: $\mathrm{R}=4-\mathrm{FC}_{6} \mathrm{H}_{4}$ 69c: $\mathrm{R}=4-\mathrm{NO}_{2} \mathrm{C}_{6} \mathrm{H}_{4}$}

图 19 化合物 69 的结构

Figure 19 Structure of compound 69

丁烯酸内酯是大部分天然产物中普遍存在的结构 单元, 刘宏民等 ${ }^{[80]}$ 将氨基二硫代甲酸酯和丁烯酸内酯 的结构进行拼合, 设计合成了 3 个系列的新型氨基二硫 代甲酸酯类化合物 70 72(图 20), 活性实验结果显示, 对于化合物 70, 当氨基部分为芐胺时, 具有广谱抗肿瘤 活性，对 5 种肿瘤细胞株(EC-9706、HeLa、PC-3、SPCA1、 $\mathrm{MCF}-7$ ) 的 $\mathrm{IC}_{50}$ 均小于 $30 \mu \mathrm{mol} / \mathrm{L}$ 系分析结果表明, 在丁 烯酸内酯的 3 位引入氨基二硫代甲酸酯侧链对化合物的 抗肿瘤活性至关重要.

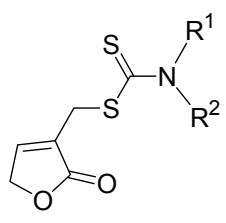

70

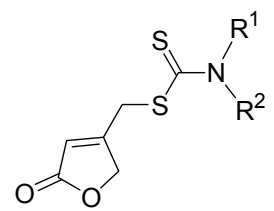

71

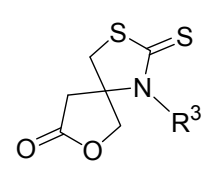

72
图 20 化合物 70 72 的结构

Figure 20 Structures of compound $\mathbf{7 0} \sim \mathbf{7 2}$

2013 年, 刘宏民课题组 ${ }^{[81 ~ 83]}$ 将 1,2,3-三氮唑结构和 氨基二硫代甲酸酯结构进行拼合, 设计、合成了两个系 列的氨基二硫代甲酸酯类化合物 73、74(图 21), 并测试 了该类化合物的抗肿瘤活性. 对于化合物 73, 当 $\mathrm{R}$ 为 $o-\mathrm{F}$ 和 $o-\mathrm{Cl}$ 时, 其对人胃癌细胞 MGC- 803 的增殖具有很 好的抑制活性, $\mathrm{IC}_{50}$ 值分别为 0.73 和 $0.49 \mu \mathrm{mol} / \mathrm{L}$. 当 $\mathrm{R}$ 为 $p$-Me 时, 其对组蛋白去甲基化酶 1 (LSD1)显示很好 的抑制活性, $\mathrm{IC}_{50}$ 为 $(2.1 \pm 0.7) \mu \mathrm{mol} / \mathrm{L}$. 对于化合物 74, 当 $\mathrm{R}^{1}$ 为 $o-\mathrm{F}, \mathrm{R}^{2} \mathrm{R}^{3} \mathrm{NH}$ 为 $\left(\mathrm{CH}_{3}\right)_{2} \mathrm{CHNH}$ 以及当 $\mathrm{R}^{1}$ 为 $p-\mathrm{F}$, $\mathrm{R}^{2} \mathrm{R}^{3} \mathrm{NH}$ 为 $\left(\mathrm{CH}_{3}\right)_{2} \mathrm{CHNH}$ 时, 化合物对人胃癌细胞 MGC-803 的抑制活性最好, $\mathrm{IC}_{50}$ 值分别为 1.62 和 0.76 $\mu \mathrm{mol} / \mathrm{L}$.

2014 年, 刘宏民等 ${ }^{[84]}$ 根据化合物 $\mathbf{7 3}$ 的构效关系研 究结果, 用香豆素替代了化合物 73 中的取代苯基单元, 设计、合成了两个系列同时含有香豆素和 1,2,3-三唑结<smiles>[R]c1cccc(Cn2cc(CSC(=S)N3CCN(C(=O)OC(C)(C)C)CC3)nn2)c1</smiles><smiles>[R][X]1ccc(Cn2cc(CSC(=S)N3CCN(C(=O)N([R])[R])CC3)nn2)cc1</smiles>

74a: $\mathrm{R}^{1}=o-\mathrm{F}, \mathrm{R}^{2} \mathrm{R}^{3} \mathrm{NH}=\left(\mathrm{CH}_{3}\right)_{2} \mathrm{CHNH}$

74b: $\mathrm{R}^{1}=p-\mathrm{F}, \mathrm{R}^{2} \mathrm{R}^{3} \mathrm{NH}=\left(\mathrm{CH}_{3}\right)_{2} \mathrm{CHNH}$

图 21 化合物 73 和 74 的结构

Figure 21 Structures of compound $\mathbf{7 3}$ and $\mathbf{7 4}$

构的氨基二硫代甲酸酯类化合物 75、76(图 22), 并评价 了该类化合物对组蛋白去甲基化酶 1 (LSD1)的抑制活 性. 研究结果显示, 在香豆素不同位置上进行取代对化 合物的活性影响很大. 当在香豆素的 3 位上进行取代时, 化合物对 LSD1 的抑制活性完全丧失. 对于化合物 75, 当 $\mathrm{R}^{1}$ 为 7-OH 时, 其对 $\mathrm{LSD} 1$ 的抑制活性最好, $\mathrm{IC}_{50}$ 值为 $0.39 \mu \mathrm{mol} / \mathrm{L}$.<smiles>[R]Cc1ccc2oc(=O)cc(Cn3cc(CSC(=S)N4CCN(C(=O)OC(C)(C)C)CC4)nn3)c2c1</smiles><smiles></smiles>

图 22 化合物 75 和 76 的结构

Figure 22 Structures of compounds $\mathbf{7 5}$ and $\mathbf{7 6}$

2015 年, 刘宏民课题组 ${ }^{[85]}$ 设计并合成了 3 个系列含 高丝氨酸内酯结构的氨基二硫代甲酸酯类化合物 77 79(图 23). 通过对这 3 个系列的化合物进行抗肿瘤活性 测试发现，与二硫代甲酸相连的基团性质对化合物的活 性影响很大. 含有疏水侧链的化合物 77 对被测试的四 种肿瘤细胞系(MGC-803、MCF-7、EC-9706、SMMC721)基本没有抑制活性，而化合物结构末端如带有取代 苯基(78), 特别是 4-氨基-查尔酮(79)骨架能显著地提高 
其抗肿瘤活性.

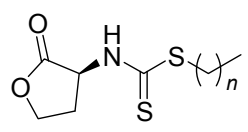

77<smiles>[R]c1ccccc1/C=C/c1ccc(NC(=O)CSC(=S)N[C@H]2CCOC2=O)cc1</smiles>

图 23 化合物 77 79 的结构

Figure 23 Structures of compounds 77 79

多年来, 我们研究组一直从事氨基二硫代甲酸酯类 化合物的合成及抗肿瘤活性方面的研究. 我们研究发 现, 哌嗪基二硫代甲酸酯类化合物具有较强的抗肿瘤活 性 ${ }^{[86]}$, 其中化合物 80(图 24)不但体外活性显著, 而且其 盐酸盐 81(图 24)对小鼠移植性肉瘤 S180、小鼠移植性 肿瘤 $\mathrm{H} 22$ 肝癌、黑色素瘤 B16、人胃癌裸鼠异种移植肿 瘤的生长均有非常明显的体内抗 S180、小鼠移植性肿瘤 H22 肝癌、黑色素瘤 B16、人胃癌裸鼠异种移植肿瘤的 生长均有非常明显的体内抗肿瘤活性, 与对照组相比有 非常显著的差异 $(P<0.001)$ 最为难能可贵的是, 它的毒 性非常低, 小鼠口服该化合物达 $10 \mathrm{~g} / \mathrm{kg}$ 时, 仍未见有小 鼠死亡(环磷酰胺的 $\mathrm{LD}_{50}$ : 小鼠口服 $\left.350 \mathrm{mg} / \mathrm{kg}\right)^{[87]}$.

为了研究该类化合物的构效关系及作用机制, 进一 步提高化合物 80 的溶解度和抗肿瘤活性, 我们以化合 物 880 为先导化合物, 对其进行深入的结构优化和构效 关系分析 ${ }^{[88,89]}$, 发现当化合物 $\mathbf{8 0}$ 的氨基部分由甲基哌 嗪改为哌嗪时, 化合物 82(图 24) 的活性和溶解度都明显 优于化合物 80, 该化合物有望开发成为一种新的抗肿 瘤药物.

我们利用液相组合化学的方法 ${ }^{[90]}$, 合成了大量的 氨基二硫代甲酸酯类化合物. 通过对所合成的化合物进 行抗肿瘤活性篮选, 发现化合物 83(图 25) 具有较强的抗 肿瘤活性, 在 $10 \mu \mathrm{mol} / \mathrm{L}$ 的浓度下, 对人肝癌细胞 Bel-7402 和人宫颈癌细胞 Hela 的抑制率分别为 $81.8 \%$ 和 $72.6 \%$. 于是, 我们以化合物 83 为先导化合物, 合成了 一系列芳基(烷基)氨基二硫代甲酸酯类化合物 $84^{[91]}$, 并 评价了该系列化合物的抗肿瘤作用. 研究发现, 该系列 化合物是一类新型的酪氨酸激酶抑制剂, 特别是化合物 84a (IC-4), 其对表皮细胞生长因子受体(EGFR)高表达 的人乳腺癌细胞 MDA-MB-468 和 MDA-MB-231 生长的 半数抑制率分别为 2.3 和 $3.6 \mu \mathrm{mol} / \mathrm{L}$, 能将 MDA-MB468 细胞的生长阻滞在 $\mathrm{G} 2 / \mathrm{M}$ 期 ${ }^{[92]}$.<smiles>CN1CCN(C(=S)SCCC(C)(c2ccccc2)c2ccccc2)CC1</smiles>

80<smiles>CN1CCN(C(=S)SCCC(C)(c2ccccc2)c2ccccc2)CC1</smiles><smiles>CC(CCSC(=S)N1CCNCC1)(c1ccccc1)c1ccccn1</smiles>

- $\mathrm{HCl}$

82

图 24 化合物 $80 、 81$ 和 82 的结构

Figure 24 Structures of $\mathbf{8 0}, \mathbf{8 1}$ and 82<smiles>[R]CCSC(=S)N([R2])CCC([R])[Y]([R])[H]</smiles>

图25 化合物 $83 、 84$ 和 $84 a$ 的结构

Figure 25 Structures of compounds 83,84 and $84 a$

为了进一步发现结构新颖、活性显著的 EGFR 抑制 剂, 我们利用药物化学中的拼合原理, 将氨基二硫代甲 酸酯基团与 4-芳氨基喹唑啉类或 3-氰基-4-芳氨基喹啉 类 EGFR 抑制剂的药效团进行拼合，设计、合成了两个 系列新型氨基二硫代甲酸酯类 EGFR 抑制剂 85、86(图 26). 抗肿瘤活性结果显示, 对于化合物 $\mathbf{8 5}^{[93]}$, 当 $\mathrm{R}^{1}$ 为 $3-\mathrm{FC}_{6} \mathrm{H}_{4} \mathrm{CH}_{2} \mathrm{O}, \mathrm{R}^{2}$ 为 $\mathrm{Cl}, \mathrm{R}^{3} \mathrm{R}^{4} \mathrm{NH}$ 为甲基哌嗪, $\mathrm{R}^{5}$ 为 $\mathrm{OMe}$ 时活性最好, 其对 EGFR 高表达的人乳腺癌细胞 MDA-MB-468 和 SK-BR-3 生长的 $\mathrm{IC}_{50}$ 分别为 3.84 和 $3.57 \mu \mathrm{mol} / \mathrm{L}$. 对于化合物 $\mathbf{8 6}^{[94]}$, 当 $\mathrm{X}$ 为 $\mathrm{N}, \mathrm{R}^{1}$ 为 $\mathrm{Cl}, \mathrm{R}^{2}$ 为 $3-\mathrm{FC}_{6} \mathrm{H}_{4} \mathrm{CH}_{2} \mathrm{O}, \mathrm{R}^{3}$ 为 $\mathrm{CH}_{2} \mathrm{CH}_{2} \mathrm{CN}$ 时活性最好, 其对 EGFR 高表达的人乳腺癌细胞 MDA-MB-468 和 SK-BR-3 生长的 $\mathrm{IC}_{50}$ 分别为 2.71 和 $2.62 \mu \mathrm{mol} / \mathrm{L}$.

除此之外, 我们在对化合物 83 进行结构优化的过 程中发现了一类新型的双(氨基二硫代甲酸)酯类化合物 $87^{[95]}$ (图 27), 当 $\mathrm{A}$ 为 3-喹啉时, 化合物 87a 具有非常显 
<smiles>[R][R]([H])=COC(N)=O</smiles>

85<smiles>[R]SC(=S)NCc1cc2c(Nc3ccc([R])c([R])c3)c[X]c([R1])c2cc1[R]</smiles>

86

图 26 化合物 85 和 86 的结构

Figure 26 Structures of compounds 85 and 86

著的抗肿瘤活性, 其对 9 种肿瘤细胞生长的 $\mathrm{IC}_{50}$ 值均小 于 $1 \mu \mathrm{mol} / \mathrm{L}$, 对肝癌细胞 HepG2 和乳腺癌细胞 MCF-7 生长的 $\mathrm{IC}_{50}$ 分别为 54 和 $23 \mathrm{nmol} / \mathrm{L}$, 很值得作为候选化 合物进行深入研究.<smiles>CN(C)C(=S)SCC(CSC(=S)N(C)C)C(=O)O</smiles>

87<smiles>CN(C)C(=S)SCC(CSC(=S)N(C)C)C(=O)c1cnc2ccccc2c1</smiles>

$87 a$
图 27 化合物 87 的结构

Figure 27 Structure of compound $\mathbf{8 7}$

\section{3 展望}

本文对氨基二硫代甲酸酯类化合物的合成及生物 活性的近期研究进展进行了综述. 从文中可以看出新的 合成方法不断涌现，合成手段不断更新，和传统方法相 比, 反应时间更短, 收率不断提高, 反应更绿色环保. 氨基二硫代甲酸酯类化合物由于其独特的分子结构, 具 有广泛的生物活性, 尤其是其作为抗肿瘤化合物已经成 为广大药物化学家研究的热点之一. 相信在不久的将 来, 氨基二硫代甲酸酯类化合物将在有机合成和药物化 学领域占有十分重要的地位.

\section{References}

[1] Ohtake, N.; Imamura, H.; Jona, H.; Kiyonaga, H.; Shimizu, A.; Moriya, M.; Sato, H.; Nakano, M.; Ushijima, R.; Nakagawa, S. Bioorg. Med. Chem. 1998, 6, 1089.

[2] Imamura, H.; Ohtake, N.; Jona, H.; Shimizu, A.; Moriya, M.; Sato, H.; Sugimoto, Y.; Ikeura, C.; Kiyonaga, H.; Nakano, M.; Nagano, R.; Abe, S.; Yamada, K.; Hashizume, T.; Morishima, H. Bioorg. Med. Chem. 2001, 9, 1571.

[3] Ohtake, N.; Imamura, H.; Kiyonaga, H.; Jona, H.; Ogawa, M.;
Okada, S.; Shimizu, A.; Moriya, M.; Sato, H.; Tominaga, Y.; Yamada, K.; Nakano, M.; Ushijima, R.; Nakagawa, S. Bioorg. Med. Chem. Lett. 1997, 7, 1617.

[4] Jang, S. Y.; Ha, Y. H.; Ko, S. W.; Lee, W.; Lee, J.; Kim, S.; Kim, Y. W.; Lee, W. K. Ha, H. Bioorg. Med. Chem. Lett. 2004, 14, 3881.

[5] Gaudernak, E.; Seipelt, J.; Triendl, A.; Grassauer, A.; Kuechler, E. J. Virol. 2002, 76, 6004.

[6] Schreck, R.; Meier, B.; Mānnel, D. N.; Drōge, W.; Baeuerle, P. A. J. Exp. Med. 1992, 175, 1181.

[7] Moellering, D.; McAndrew, J.; Jo, H.; Darley-Usmar, V. M. Free Radic. Biol. Med. 1999, 26, 1138.

[8] Meyer, M.; Schreck, R.; Baeuerle, P. A. EMBO J. 1993, 12, 2005.

[9] Schenk, H.; Klein, M.; Erdbrügger, W.; Dröge, W.; SchulzeOsthoff, K. Proc. Natl. Acad. Sci. U. S. A. 1994, 91, 1672.

[10] Brewer, C. Alcohol Alcohol. 1993, 28, 383.

[11] Hidaka, S.; Funakoshi, T.; Shimada, H.; Tsuruoka, M.; Kojima, S. J. Appl. Toxicol. 1995, 15, 267.

[12] Tandon, S. K.; Singh, S.; Jain, V. K.; Prasad, S. Fundam. Appl. Toxicol. 1996, 31, 141.

[13] Gerhäuser, C.; You M.; Liu, J. F.; Moriarty, R. M.; Hawthorne, M.; Mehta, R. G.; Moon, R. C.; Pezzuto, J. M. Cancer Res. 1997, 57, 272.

[14] Gaspari, P.; Banerjee, T.; Malachowski, W P.; Muller, A J.; Prendergast, G C.; DuHadaway, J.; Bennett, S.; Donovan, A M. J. Med. Chem. 2006, 49, 684.

[15] Burke, Jr., T. R.; Bajwa, B. S.; Jacobson, A. E.; Rice, K. C.; Streaty, R. A.; Klee, W. A. J. Med. Chem. 1984, 27, 1570.

[16] Walter, W.; Bode, K.-D. Angew. Chem., Int. Ed. Engl. 1967, 6, 281.

[17] (a) Lieber, E.; Orlowski, R. O. J. Org. Chem. 1957, 22, 88.

(b) Lee, A. W. M.; Chan, W. H.; Wong, H. C.; Wong, M. S. Synth. Commun. 1989, 19, 547.

(c) Degani, I.; Fochi, R. Synthesis 1978, 365.

[18] Azizi, N.; Khajeh, M.; Hasani, M.; Dezfooli, S. Tetrahedron Lett. 2013, 54, 5407.

[19] (a) Salvatore, R. N.; Sahab, S.; Jung, K. W. Tetrahedron Lett. 2001, 42, 2055.

(b) Nagle, A. S.; Salvatore, R. N.; Cross, R. M.; Kapxhiu, E. A.; Sahab, S.; Yoon, C. H.; Jung, K. W. Tetrahedron Lett. 2003, 44, 5695.

[20] Fox. D. L.; Ruxer, J. T.; Oliver, J. M.; Alford, K. L.; Salvatore, R. N. Tetrahedron Lett. 2004, 45, 401.

[21] (a) Li, R.-T.; Ding, P.-Y.; Han, M.; Cai, M.-S. Synth. Commun. 1998, 28, 295.

(b) Li, R. T.; Cai, M. S. Synth. Commun. 1999, 29, 65 .

(c) Ge, Z. M.; Li, R. T.; Cheng, T. M. Synth. Commun. 1999, 29, 3191.

(d) Li, R. T.; Ge, Z. M.; Cheng, T. M.; Cai, M. S. Chem. J. Chin. Univ. 1999, 20, 1897 (in Chinese).

(李润涛, 葛泽梅, 程铁明, 蔡孟深, 高等学校化学学报, 1999, 20,1897.)

(e) Cui, J. L.; Ge, Z. M.; Cheng, T. M.; Li, R. T. Synth. Commun. 2003, 33, 1969.

(f) Guo, B. G.; Ge, Z. M.; Cheng, T. M.; Li, R. T. Synth. Commun. 2001, 31, 3021.

[22] Han, F. B.; Ge, Z. M.; Cheng, T. M.; Li, R. T. Synlett 2009, 648.

[23] Xia, S.; Wang, X.; Ge, Z. M.; Cheng, T. M.; Li, R. T. Tetrahedron 2009, 65, 1005.

[24] Sha, Q.; Wei, Y. Y. Org. Biomol. Chem. 2013, 11, 5615.

[25] Hemantha, H. P.; Sureshbabu, V. V. Tetrahedron Lett. 2009, 50, 7062.

[26] (a) Azizi, N.; Aryanasab, F.; Torkiyan, L.; Ziyaei, A.; Saidi, M. R. J. Org. Chem. 2006, 71, 3634. 
(b) Ziyaei-Halimjani, A.; Saidi, M. R. Can. J. Chem. 2006, 84, 1515 .

(c) Azizi, N.; Aryanasab, F.; Torkiyan, L.; Saidi, M. R. Synth. Commun. 2011, 41, 94.

[27] Bhadra, S.; Saha, A.; Ranu, B. C. Green Chem. 2008, 10, 1224

[28] Chatterjee, T.; Bhadra, S.; Ranu, B. C. Green Chem. 2011, 13, 1837

[29] Yadav, L. D. S.; Patel, R.; Srivastava, V. P. Tetrahedron Lett. 2009, 50,1335 .

[30] Halimehjani, A. Z.; Marjani, K.; Ashouri, A. Green Chem. 2010, $12,1306$.

[31] Karmakar, B.; Banerji, J. Tetrahedron Lett. 2011, 52, 6584.

[32] Azizi, N.; Gholibeglo, E.; Nayeri, S. D. Monatsh. Chem. 2012, 143, 1171.

[33] Ranu, B. C.; Saha, A.; Banerjee, S. Eur. J. Org. Chem. 2008, 519.

[34] (a) Azizi, N.; Aryanasab, F.; Saidi, M. R. Org. Lett. 2006, 8, 5257.

(b) Azizi, N.; Pourhasan, B.; Aryanasab, F.; Saidi, M. R. Synlett 2007, 1239.

(c) Azizi, N.; Ebrahimi, F.; Aakbari, E.; Aryanasab, F.; Saidi, M. R. Synlett 2007, 2797.

(d) Aryanasab, F.; Saidi, M. R. Monatsh. Chem. 2014, 145, 521.

[35] Jana, M.; Misra, A. K. Synlett 2012, 2789.

[36] Guntreddi, T.; Vanjari, R.; Singh, K. N. Tetrahedron 2014, 70, 3887.

[37] Chaturvedi, D.; Ray, S. Tetrahedron Lett. 2006. 47,1307.

[38] Saha, A.; Baig, R. B. N.; Leazer, J.; Varma, R. S. Chem. Commun. 2012, 48, 8889 .

[39] Payra, S.; Saha, A.; Peng, R.; Wu, C-M.; Koodali, R. T.; Banerjee, S. Tetrahedron Lett. 2015, 56, 1609.

[40] Brewer, C. Alcohol Alcohol. 1993, 28, 383.

[41] Thorn, G. D.; Ludwig, R. A. Elsevier Amsterdam 1962.

[42] Lutz, L. M.; Glende, E. A.; Recknagel, R. O. Biochem. Pharmacol. 1973, 22, 1729.

[43] Bartoli, G. M.; Müller, A.; Cadenas, E.; Sies, H. FEBS Lett. 1983, 164, 371.

[44] Zanocco, A. L.; Pavez, R.; Videla, L. A.; Lissi, E. A. Free Radical Biol. Med. 1989, 7, 151.

[45] Schreck, R.; Meier, B.; Mānnel, D. N.; Drōge, W.; Baeuerle, P. A. J. Exp. Med. 1992, 175, 1181 .

[46] Moellering, D.; McAndrew, J.; Jo, H.; Darley-Usmar, VM. Free Radical Biol. Med. 1999, 26, 1138.

[47] Meyer, M.; Schreck, R.; Baeuerle, P. A. EMBO J. 1993, 12, 2005.

[48] Schenk, H.; Klein, M.; Erdbrügger, W.; Dröge, W. Proc. Natl. Acad. Sci. U. S. A. 1994, 91, 1672.

[49] Schreck, R.; Meier, B.; Männel, D. N.; Dröge, W.; Baeuerle, P. A. J. Exp. Med. 1992, 175, 1181.

[50] Wolfe, J. T.; Ross, D.; Cohen, G. M. FEBS Lett. 1994, 352, 58.

[51] Bessho, R.; Matsubara, K.; Kubota, M.; Kuwakado, K.; Hirota, H.; Wakazono, Y.; Lin, Y. W.; Okuda, A.; Kawai, M.; Nishikomori, R.; Heike, T. Biochem. Pharmacol. 1994, 48, 1883.

[52] Gaudernak, E.; Seipelt, J.; Triendl, A.; Grassauer, A.; Kuechler, E. J. Virol. 2002, 76, 6004 .

[53] Ohtake, N.; Imamura, H.; Kiyonaga, H.; Jona, H.; Ogawa, M.; Okada, S.; Shimizu, A.; Moriya, M.; Sato, H.; Tominaga, Y.; Yamada, K.; Nakano, M.; Ushijima, R.; Nakagawa, S. Bioorg. Med. Chem. Lett. 1997, 7, 1617.

[54] Imamura, H.; Ohtake, N.; Jona, H.; Shimizu, A.; Moriya, M.; Sato, H.; Sugimoto, Y.; Ikeura, C.; Kiyonaga, H.; Nakano, M.; Nagano, R.; Abe, S.; Yamada, K.; Hashizume, T.; Morishima, H. Bioorg. Med. Chem. 2001, 9, 1571.

[55] Horita, Y.; Takii, T.; Chiba, T.; Kuroishi, R.; Maeda, Y.; Kurono, Y.; Inagaki, E.; Nishimura, K.; Yamamoto, Y.; Abe, C.; Mori, M.; Onozaki, K. Bioorg. Med. Chem. Lett. 2009, 19, 6313.
[56] Ren, J. L.; Zhang, E.; Ye, X. W.; Wang, M. M.; Yu, B.; Wang, W. H.; Guo, Y. Z.; Liu, H. M. Bioorg. Med. Chem. Lett. 2013, 23, 4154.

[57] Zou, Y.; Yu, S. C.; Li, R. W.; Zhao, Q. J.; Li, X.; Wu, M. C.; Huang, T.; Chai, X. X.; Hu, H. G.; Wu, Q. Y. Eur. J. Med. Chem. 2014, 74, 366.

[58] Bala, V.; Jangir, S.; Mandalapu, D.; Gupta, S.; Chhonker, Y. S.; Lal, N.; Kushwaha, B.; Chandasana, H.; Krishna, S.; Rawat, K.; Maikhuri, J. P.; Bhatta, R. S.; Siddiqi, M. I.; Tripathi, R.; Gupta, G.; Sharma, V. L. Bioorg. Med. Chem. Lett. 2015, 25, 881.

[59] (a) Altintop, M. D.; Özdemir, A.; Kaplancikli, Z. A.; Turan-Zitouni, G.; Temel, H. E.; Çiftçi, G. A. Arch. Pharm. Chem. Life Sci. 2013, $346,189$.

(b) Altıntop, M. D.; Gurkan-Alp, A. S.; Özkay, Y.; Kaplancıkli, Z. A. Arch. Pharm. Chem. Life Sci. 2013, 346, 571.

[60] Mehta, R. G.; Liu, J.; Constantinou, A.; Thomas, C. F.; Hawthorne, M.; You, M.; Gerhüser, C.; Pezzuto, J. M.; Moon, R. C.; Moriarty, R. M. Carcinogenesis 1995, 16, 399.

[61] Pedras, M. S.; Loukaci, A.; Okanga, F. I. Bioorg. Med. Chem. Lett. 1998, 8, 3037.

[62] Mehta, R. G.; Liu, J.; Constantinou, A.; Hawthorne, M.; Pezzuto, J. M.; Moon, R. C.; Moriarty, R. M. Anticancer Res. 1994, 14, 1209.

[63] Donovan, A. M.; Bennett, S.; DuHadaway, J.; Prendergast, G. C.; Muller, A. J.; Malachowski, W. P.; Banerjee, T.; Gaspari, P. J. Med. Chem. 2006, 49, 684.

[64] Zhang, Y.; Talalay, P.; Cho, C. G.; Posner, G. H. Proc. Natl. Acad. Sci. U. S. A. 1992, 89, 2399.

[65] Ye, L. X.; Zhang, Y. S. Carcinogenesis 2001, 22, 1987.

[66] Gerhäuser, C.; You M.; Liu, J. F.; Moriarty, R. M.; Hawthorne, M.; Mehta, R. G.; Moon, R. C.; Pezzuto, J. M. Cancer Res. 1997, 57, 272.

[67] Hirschelman, W. H.; Song, L. S.; Park, E. J.; Tan, Y.; Yu, R.; Hawthorne, M.; Mehta, R. G.; Grubbs, C. J.; Lubet, R. A.; Moriarty, R. M.; Pezzuto, J. M. $224^{\text {th }}$ ACS National Meeting: Division of Medicinal Chemistry, Boston, 2002, p. 98.

[68] Scozzafava, A.; Supuran, C. T. Bioorg. Med. Chem. Lett. 2000, 10, 1117.

[69] Scozzafava, A.; Mastrolorenzo, A.; Supuran, C. T. Bioorg. Med. Chem. Lett. 2000, 10, 1887.

[70] Özlen, G.; Salman, A. Bioorg. Med. Chem. 2006, 14, 7804.

[71] Zahran, M. A.-H.; Salemb, T. A. R.; Samaka, R. M.; Agwa, H. S.; Awad, A. R. Bioorg. Med. Chem. 2008, 16, 9708.

[72] Huang, W.; Ding, Y.; Miao, Y.; Liu, M. Z.; Yang, G. F. Eur. J. Med. Chem. 2009, 44, 3687.

[73] Qian, Y.; Ma, G. Y.; Yang, Y.; Chen, K.; Zheng, Q. Z.; Mao, W. J.; Shi, L.; Zhao, J.; Zhu, H. L. Bioorg. Med. Chem. 2010, 18, 4310.

[74] Bacharaju, K.; Jambula, S. R.; Sivan, S.; Tangeda, S. J.; Manga, V. Bioorg. Med. Chem. Lett. 2012, 22, 3274.

[75] Jangir, S.; Bala, V.; Lal, N.; Kumar, L.; Sarswat, A.; Kumar, A.; Hamidullah; Saini, K. S.; Sharma, V.; Verma, V.; Maikhuri, J. P.; Konwar, R.; Gupta, G.; Sharma, V. L. Eur. J. Med. Chem. 2014, 85, 638.

[76] Cao, S. L.; Feng, Y. P.; Jiang, Y. Y.; Liu, S. Y.; Ding, G. Y.; Li, R. T. Bioorg. Med. Chem. Lett. 2005, 15, 1915.

[77] Cao, S. L.; Feng, Y. P.; Zheng, X. L.; Jiang, Y. Y.; Jiang, Y. Y.; M.; Wang, Y.; Xu, M. Arch. Pharm. Chem. Life Sci. 2006, 339, 250.

[78] Cao, S. L.; Wang, Y.; Zhu, L.; Liao, J.; Guo, Y. W.; Chen, L. L.; Liu, H. Q.; Xu, X. Z. Eur. J. Med. Chem. 2010, 45, 3850.

[79] Cao, S. L.; Han, Y.; Yuan, C. Z.; Wang, Y.; Xiahou, Z. K.; Liao, J.; Gao, R. T.; Mao, B. B.; Zhao, B. L.; Li, Z. F.; Xu, X. Z. Eur. J. Med. Chem. 2013, 64, 401.

[80] Wang, X. J.; Xu, H. W.; Guo, L. L.; Zheng, J. X.; Xu, B.; Guo, X.; 
Zheng, C. X.; Liu, H. M. Bioorg. Med. Chem. Lett. 2011, 21, 3074.

[81] Duan, Y. C.; Zheng, Y.. C.; Li, X. C.; Wang, M. M. Ye, X. W.; Guan, Y. Y.; Liu, G. Z.; Zheng, J. X.; Liu, H. M. Eur. J. Med. Chem. 2013, 62, 11.

[82] Zheng, Y. C.; Duan, Y. C.; Ma, J. L.; Xu, R. M.; Zi, X. L.; Lv, W. L.; Wang, M. M.; Ye, X. W.; Zhu, S.; Mobley, D.; Zhu, Y. Y.; Wang, J. W.; Li, J. F.; Wang, Z. R.; Zhao, W.; Liu, H. M. J. Med. Chem. 2013, 56, 8543 .

[83] Duan, Y. C.; Zheng, Y. C.; Li, X. C.; Wang, M. M.; Ye, X. W.; Guan, Y. Y.; Liu, G. Z.; Zheng, J. X.; Liu, H. M. Eur. J. Med. Chem. 2013, 64, 99 .

[84] Ye, X. W.; Zheng, Y. C.; Duan, Y. C.; Wang, M. M.; Yu, B.; Ren, J. L.; Ma, J. L.; Zhang, E.; Liu, H. M. Med. Chem. Commun. 2014, 5, 650.

[85] Ren, J. L.; Zhang, X. Y.; Yu, B.; Wang, X. X.; Shao, K. P.; Zhu, X. G.; Liu, H. M. Eur. J. Med. Chem. 2015, 93, 321.

[86] Li, R. T.; Cheng, T. M.; Cui, J. R. WO 2002096869, 2002 [Chem. Abstr. 2003, 138, 170256].

[87] Guo, W.; R, F. X.; Wang, R. Q.; Cui, J. R.; Li, R. T.; Cheng, T. M.; Ge, Z. M. Chin. J. Clin. Pharmacol. Ther. 2004, 9(1), 59 (in
Chinese).

(郭维, 再福香, 王瑞卿, 崔景荣, 李润涛, 程铁明, 葛泽梅, 中 国临床药理学与治疗学, 2004, 9(1), 59.)

[88] Hou, X. L.; Ge, Z. M.; Wang, T. M.; Guo, W.; Cui, J. R.; Cheng, T. M.; Lai, C. S.; Li, R. T. Bioorg. Med. Chem. Lett. 2006, 16, 4212.

[89] Hou, X. L.; Ge, Z. M.; Wang, T. M.; Guo, W.; W, J.; Cui, J. R.; Lai, C. S.; Li, R. T. Arch. Pharm. Chem. Life Sci. 2011, 11, 320.

[90] Wei, J. M.S. Thesis, Peking University, Beijing, 2002 (in Chinese). (卫军, 硕士论文, 北京大学, 北京, 2002.)

[91] Li, R. T.; Ge, Z. M.; Cui, J. R.; Sun, X. Y.; Wang, Z. Q.; Yan, X. CN 102234271, 2010 [Chem. Abstr. 2011, 155, 562474].

[92] Li, Y. B.; Wang, Z. Q.; Yan, X.; Chen, M. W.; Bao, J. L.; Wu, G. S.; Ge, Z. M.; Zhou, D. M.; Wang, Y. T.; Li, R. T. Cancer Lett. 2013, $340,88$.

[93] Li, R. D.; Zhang, X.; Li, Q. Y.; Ge, Z. M.; Li, R. T. Bioorg. Med. Chem. Lett. 2011, 21, 3637.

[94] Zhang, X.; Li, R. D.; Qiao, K.; Ge, Z. M.; Zhang, L. R.; Cheng, T. M.; Li, R. T. Arch. Pharm. Chem. Life Sci. 2013, 346, 44.

[95] Li, R. D.; Wang, H. L.; Li, Y. B.; Wang, Z. Q.; Wang, X.; Wang, Y. T.; Ge, Z. M.; Li, R. T. Eur. J. Med. Chem. 2015, 93, 381.

(Qin, X.) 